\title{
Cutaneous Carcinosarcoma: A Small Case Series and Review of the Literature of a Rare Skin Tumor
}

\author{
Ethan Y. Song ${ }^{1}$, Sean J. Wallace ${ }^{2}$, Hina Sheikh ${ }^{3}$, Randolph Wojcik Jr. ${ }^{2}$, Chetan Nayak ${ }^{4}$ \\ 1. Plastic and Reconstructive Surgery, University of South Florida Morsani College of Medicine, Tampa, USA 2. Division \\ of Plastic \& Reconstructive Surgery, Lehigh Valley Health Network, Allentown, USA 3. Pathology, Health Network \\ Laboratories, Allentown, USA 4. Otolaryngology, Lehigh Valley Health Network, Allentown, USA
}

Corresponding author: Ethan Y. Song, yichen.song94@gmail.com

\begin{abstract}
Carcinosarcomas are composed of epithelial and mesenchymal elements and primarily present within visceral organs. Despite being potentially aggressive, they are a rare diagnosis in the skin, and few manifestations have been reported to date. In this report, we describe two separate cases of carcinosarcoma presenting as nonhealing scalp wounds. Patient A: a 57-year-old male with a nonhealing skin lesion of ten years successfully treated with wide-local excision and local ortichochea flap reconstruction. Patient B: a 75 -year-old female that presented with a painless, slow-growing hemorrhagic mass of 7 years invading the skull and dura ultimately requiring craniectomy and free-tissue transfer with anterolateral thigh flap. Cutaneous carcinosarcomas have more favorable outcomes due to low metastatic rates likely due to earlier detection, but delayed presentation can be fatal. Histopathological analysis is critical for determining diagnosis and prognosis. Adequate reconstruction after wide base excision varies and follows the reconstructive ladder/elevator ranging from primary closure up through free-tissue transfer. With cutaneous manifestations of carcinosarcoma seldom reported in the literature, it is our hope that reporting unusual instances such as this will raise awareness and allow for earlier diagnoses, treatments, and reconstructions.
\end{abstract}

Categories: Dermatology, Pathology, Plastic Surgery

Keywords: cutaneous carcinosarcoma, sarcomatoid carcinoma, skin cancer

\section{Introduction}

Cutaneous carcinosarcoma is a rare, biphasic cancer composed of epithelial and mesenchymal tissues that were first described in 1972 [1]. Carcinosarcomas have been primarily associated with visceral organs and previously described most commonly in the uterus, ovary, lung, gastrointestinal tract, prostate, thyroid, and testis [2-4]. Secondary to their late presentations viscerally, they tend to have high malignant potential and poor prognoses. It is not feasible to distinguish cutaneous carcinosarcoma from other similar-appearing skin cancers based on clinical appearance alone. Diagnosis requires clinical suspicion and subsequent

Received 07/13/2020

Review began 07/18/2020 Review ended 07/29/2020 Published 08/05/2020

\section{() Copyright 2020}

Song et al. This is an open access article distributed under the terms of the Creative Commons Attribution License CC-BY 4.0., which permits unrestricted use, distribution, and reproduction in any medium, provided the original author and source are credited. histopathologic sampling of concerning tissue. Histomorphologic biphasic cell populations of the epithelial and mesenchymal components showing differential immunoreactivity with epithelial element being positive for keratin markers and p63 while mesenchymal elements showing reactivity for markers such as CD10, CD99 and variable (weaker to absent) epithelial marker expression are defining diagnostic features [5]. Negative prognostic factors include aggressive histologic subtypes such as squamous cell component, earlier age of presentation, tumor size $>2 \mathrm{~cm}$, and nodal involvement [6,7].

In this report, we describe two unusual cases of carcinosarcoma presenting on the scalp. We present the clinical, surgical, and histopathological features, discuss the treatment and reconstructive methods based on tissue deficit, and finally review the published medical literature on non-visceral, cutaneous carcinosarcomas.

\section{Case Presentation}

\section{Patient A}

A 57-year-old male presented with a painless mass at the scalp vertex that had been present for 10 years' time. Past medical history was most significant for BRCA2 mutation, squamous cell carcinoma (SCC) of the eyelid status-post excision and radiation therapy, and prostate cancer status-post radical prostatectomy and radiation therapy. Family history was significant for cancers including lymphoma, prostate, pancreatic, skin, ovarian, breast, and thyroid.

On exam, he had a palpable, tender, non-mobile 2 x $2 \mathrm{~cm}$ cutaneous mass at scalp vertex (Figure $1 \mathrm{~A}$ ). A shave biopsy revealed biphasic cytology: one component with moderate-to-poorly differentiated squamous carcinoma, connected to and emanating from the epidermis, and the other more pleomorphic undifferentiated spindled and histiocytoid cells with atypical mitotic figures, representing a sarcomatous 


\section{Cureus}

component. The immunoprofile mirrored morphologic differences in these populations (carcinomatous areas were strongly positive for keratins, CK5/6 and AE1/AE3 and also p63; sarcomatous areas were strongly positive for CD68, CD163 and show only scattered staining of single cells with AE1/AE3). Based on histologic and immunohistochemical observations, the tumor was determined to be a carcinosarcoma (Figure $2 \mathrm{~A}, \mathrm{~B}$ ).
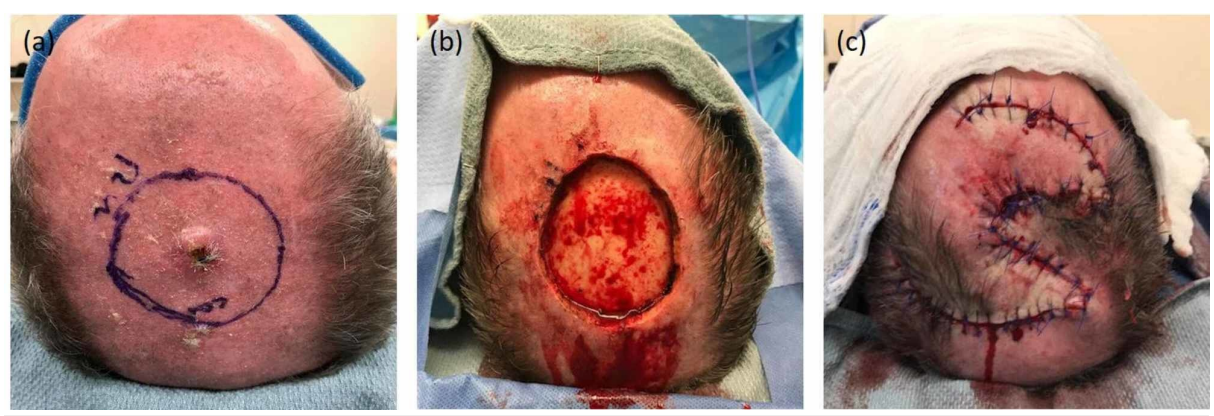

FIGURE 1: Patient A: (A) pre-excision, (B) post-excision defect illustrating exposed bone devoid of pericranium, and $(C)$ reconstructed scalp wound with local tissue rearrangement (ortichochea flap)
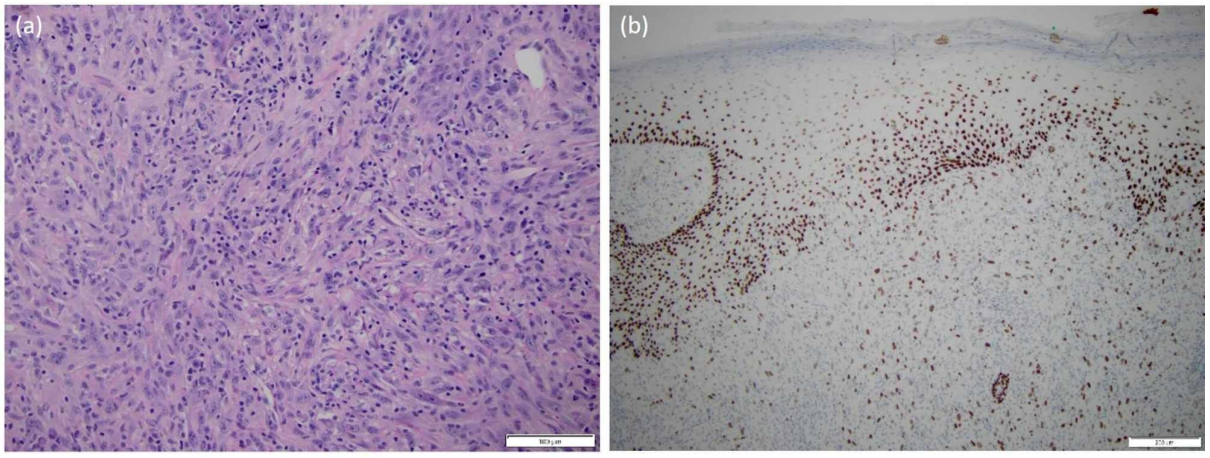

FIGURE 2: Patient A: (A) a representative routine H\&E section at 20x showing spindled and undifferentiated histomorphology classically seen in carcinosarcoma and $(B)$ a representative immunohistochemical stain at 10x showing variable p63 positivity targeting the diagnosis of carcinosarcoma

H\&E, hematoxylin and eosin

Radiological evaluation was negative for the involvement of the cervical or suboccipital lymph nodes. He underwent a wide local excision with frozen section. The surgical defect measured $5.5 \times 5.5 \mathrm{~cm}$ and had exposed skull devoid of pericranium (Figure $1 B$ ). To provide adequate and durable well-vascularized soft tissue coverage, an ortichochea local tissue rearrangement was designed utilizing three separate rotational flaps equally positioned around the defect (Figure 1C). Final pathology revealed carcinosarcoma with negative margins.

At outpatient follow-up through 12 months, he had no evidence of recurrence, metastasis, or woundhealing complications.

\section{Patient B}

A 75-year old female with no significant past medical history presented for evaluation of a painless, nonhealing wound on her scalp of seven years' time. Associated symptoms included recent and rapid significant unexplained weight loss, as well as anemia.

On examination, she had a $7 \times 6 \mathrm{~cm}$ ulcerating, fungating mass at the scalp occiput extending towards the right parietal region, as well as a palpable lymph node along the anterior border of the right sternocleidomastoid muscle. A punch biopsy revealed mixed basal cell carcinoma (BCC) along with a poorlydifferentiated malignant component of spindled, epithelioid, and pleomorphic cytology closely intermingled 


\section{Cureus}

with the BCC cells. On immunostains, CD99 showed extensive positivity of BCC and poorly differentiated tumor cells. These poorly differentiated cells were positive for vimentin, showed focal staining for smooth muscle actin, and were negative for cytokeratin AE1/AE3, cytokeratin 903, S100, MART and CD45. Poorly differentiated malignant cells were negative for HMB45, CD3, CD20, CD34, synaptophysin and cytokeratin 20, further excluding the possibility of co-existent melanoma, lymphoma, angiosarcoma and Merkel cell carcinoma. The histopathologic morphology and immunoprofile supported a diagnosis of basal cell carcinosarcoma (Figure 3A, B).
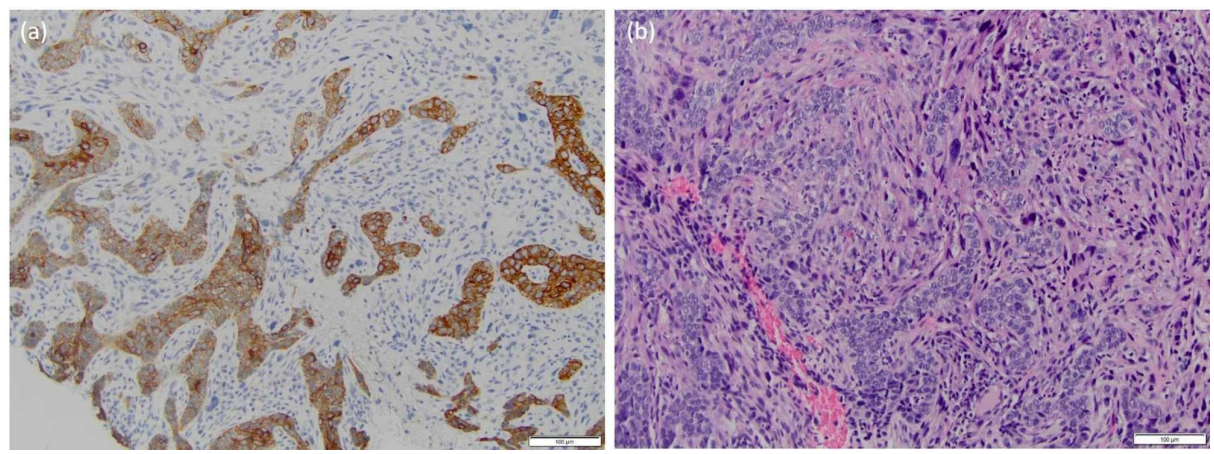

FIGURE 3: Patient B: (A) basal cell carcinosarcoma showing biphasic cytology at 20x and (B) differential cytokeratin immunostaining at 20x magnification with sarcomatoid cells staining negative

CT of the head illustrated an underlying 3.7 x $3.8 \mathrm{~cm}$ component involving skull and dura (Figure $4 A-C)$. She underwent pre-operative chemotherapy with Vismodegib. At surgical extirpation, a $7 \mathrm{~cm}$ hemorrhagic tumor was removed. The defect measured $12 \times 7 \mathrm{~cm}$. Within the wound bed, there was skull devoid of pericranium, as well as a transcranial defect with exposed brain. To provide adequate and durable well-vascularized soft tissue coverage, an anterior lateral thigh free-tissue transfer was performed. Final pathology confirmed carcinosarcoma with negative margins.
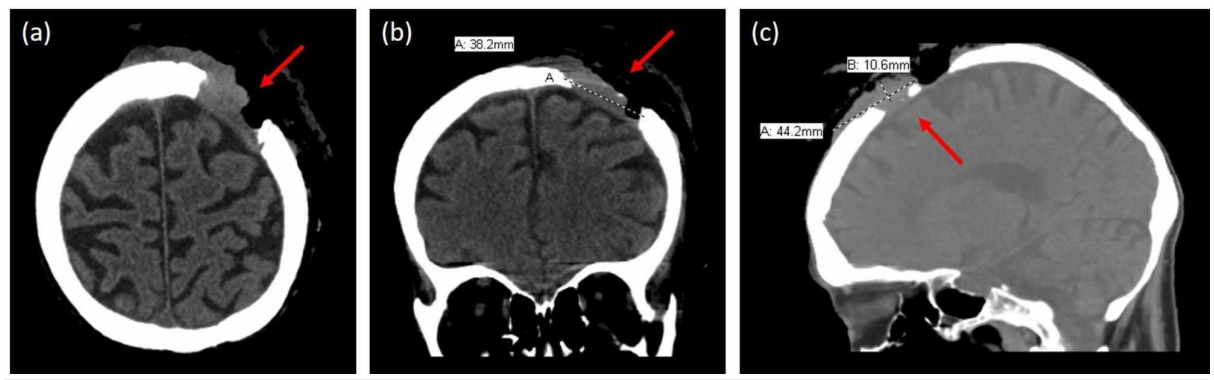

FIGURE 4: Patient B: Computed tomography scan in the (A) axial, (B) coronal and $(\mathrm{C})$ sagittal views illustrating cranial bone and brain involvement of carcinosarcoma

Initial outpatient follow-up revealed her to be recovering well; however, within 30 days, she was hospitalized due to a fall and was found to have a recurrence of the carcinosarcoma with extension into the brain on trauma imaging. She underwent a second palliative surgery and received postoperative radiation therapy, yet her condition continued to deteriorate, and she ultimately succumbed to her disease.

\section{Discussion}

Rarely reported as a primary tumor of the skin with only around 130 total cases documented, carcinosarcomas have been associated with better outcomes secondary to earlier diagnosis. Worldwide, few cases of cutaneous carcinosarcoma have been reported in the medical literature. A review of PubMed Medline involving reports of carcinosarcoma utilizing search terms (cutaneous carcinosarcoma [Title/abstract] AND carcinosarcoma of the skin [Title/abstract]) AND ((skin OR subcutaneous OR cutaneous OR superficial)) revealed only 65 articles, with the majority as case reports and/or small case series (Table 1). 


\section{Cureus}

306185132019 Cutaneous Carcinosarcoma: a Clinicopathologic and Immunohistochemical Analysis of 11 Korean Cases

307895182019 Cutaneous Carcinosarcoma of the Medial Canthus Discovered on Mohs Debulk Analysis

296666952018 Primary Cutaneous Carcinosarcoma: A cutaneous neoplasm with an exceptional presentation.

299545912018 Pilomatrical Carcinosarcoma: Report of a Case with Comparative Genomic Hybridizsation Analysis

302833132018 A Case of Primary Cutaneous Basal Cell Carcinoma

299545912018 Trichoblastic Carcinosarcoma of the skin: A Case Report and Literature Review

296247002018 Sarcomatoid Pilomatrix Carcinoma

294840022018 Giant Pendulous Carcinosarcoma - Squamous cell Carcinoma Type - of the Leg - A Case Report and Review of the Literature

292931242018 Cutaneous Metaplastic Carcinoma: Report of a Case With Sebaceous Differentiation

284810302017 Pilomatrical Carcinosarcoma of the Cheek: Immunohistochemica and Molecular Analysis of Beta-Catenin

284527202017 Carcinosarcoma of Skin (sarcomatoid carcinoma) - A Rare Non-Melanoma Skin Cancer (Case Review)

277306572017 Cutaneous Carcinosarcoma: A Series of Six Cases and a Review of the Literature

279102772016 Aggressive Growth of an Incompletely Excised Primary Cutaneous Basal Cell Carcinosarcoma on the Scalp: A case Report

272059052016 A Growing Scalp Nodule

271946742016 Trichoblastic Carcinosarcoma: An Authentic Cutaneous Carcinosarcoma

262724772016 Squamous cell carcinoma with osteoclast-like giant cells: a morphologically heterologous group including carcinosarcoma and squamous cell carcinoma with stromal changes

271639272016 Exophytic Scalp Tumor

257756192015 Primary Cutaneous Carcinosarcoma of the Basal Cell Subtype Should be Treated as High-Risk BCC

262684722015 Cutaneous Basal Cell Carcinosarcoma: Case Report and Literature Review

253287372014 Cutaneous Carcinosarcoma with Metastasis to the Parotid Gland

249786452014 Cutaneous Carcinosarcoma Arising from a Burn Scar

246989342014 Basal Cell Carcinosarcoma: A Report of 4 Cases and Review of the Literature

243432112014 Carcinosarcoma: A Primary Cutaneous Tumor with Biphasic Differentiation

243356432014 Basal Cell Carcinosarcoma with PTCH1 Mutations in both Epithelial and Sarcomatoid Primary Tumor Components and in the Sarcomatoid metastasis

232056222013 Primary Cutaneous Carcinosarcoma of the Shoulder: Case Report with Literature Review

229608382012 Primary Cutaneous Sarcomatoid Carcinoma

224398852012 Sarcomatoid Carcinoma of the Hand: A Clinical Case with an Aggressive and Uncommon Presentation

216589822012 Merkel Cell Carcinosarcoma: Merkel Cell Carcinoma with Embyronal Rhabdomyosarcoma-like Component

217885652011 Carcinosarcoma Derived from Nevus Sebaceus

214647222011 Carcinosarcoma Ex Eccrine Spiradenoma of the Vulva: Report of the First Case

205980632010 Carcinosarcoma of the Vulva: A Case Report

205701082010 Metastatic Cutaneous Carcinosarcoma to the Tongue

201488372010 Primary Cutaneous Carcinosarcoma: Dermatoscopic and Immunohistochemical Features

222826732010 Primary Carcinosarcoma of the Skin

196845122009 Cutaneous Carcinosarcoma with Myoepothelial Differentiation: Immunohistochemical and Cytogenetic Analysis of a Case Presenting in an Unusual location

187939372008 Basal Cell Carcinoma with a Sarcomatous Component (carcinosarcoma): a series of 5 cases and review of the literature 


\section{Cureus}

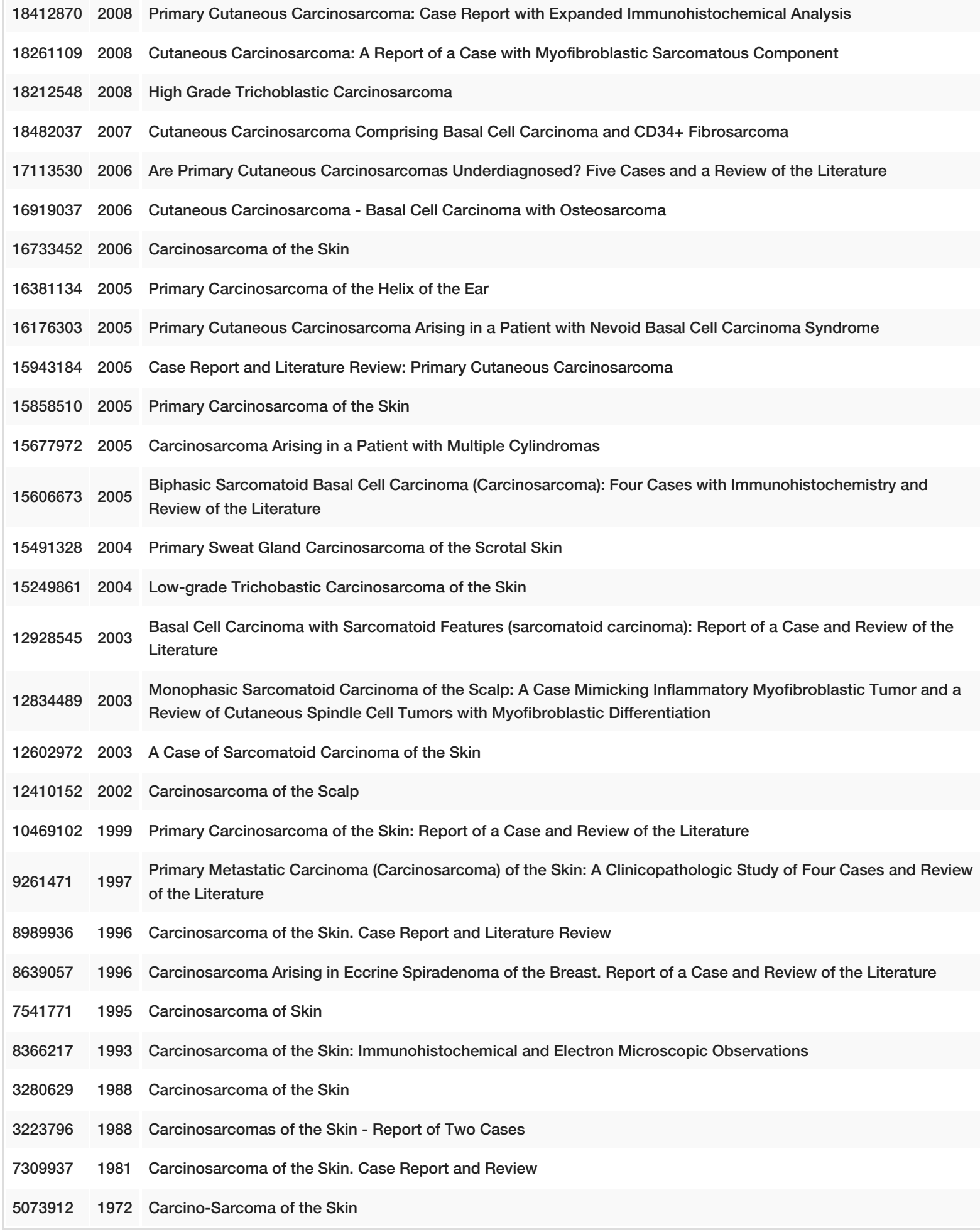

TABLE 1: Review of PubMed Medline literature involving case reports and case series of nonvisceral carcinosarcomas

Cutaneous carcinosarcoma tend to be found in adults in chronically sun-exposed regions after the fifth decade of life with a male predominance 1.7:1 [5,8,9]. These tumors are known for their biphasic nature, rapid rate of growth, ulceration, and exophytic-appearance that can be misleading to even the mostexperienced clinicians. Several histologic forms of carcinosarcoma of the skin have been described, including basal cell, squamous cell, pilomatrical, and trichoblastic carcinosarcoma [10-12]. As opposed to adnexally derived carcinosarcomas, epidermally derived carcinosarcomas are reported to have a better fiveyear survival rates, with disease-free survival of $70 \%$ compared to $25 \%$, respectively [6]. However, the survival rates of cutaneous carcinosarcomas are not as high as that of conventional BCC and SCC, of which 
There are currently several existing hypotheses to explain the development of carcinosarcomas, which include divergent differentiation of a single progenitor cell into malignant epithelial and mesenchymal populations (monoclonal hypothesis), origin from two separate progenitor cells (multiclonal hypothesis), and a collision tumor of two unique neoplasms [6,14]. At present, the monoclonal hypothesis is the most accepted, with a study by Bigby et al. reporting that sarcomatous components have been recognized as a metaplastic carcinoma of epithelial components [7].

Since carcinosarcomas are a biphasic malignancy, the epithelial components can be a mix of BCC due to mutations in PTCH1, p53, p63, and p13 gene mutations and SCC from point mutations in p53 [5]. The sarcomatous component is now accepted to be the result of divergent mesenchymal differentiation, or metaplasia, of the epithelial component [14]. The mesenchymal constituents have been described as either homologous (site-appropriate) or heterologous (site-inappropriate). Homologous forms include leiomyosarcoma and pleomorphic sarcoma, while heterologous forms include osteosarcoma, chondrosarcoma, and rhabdomyosarcoma [3]. More than one subtype can coexist in the same tumor.

To add to existing literature, we report two cases of carcinosarcoma of the scalp. Interestingly, both patients had nonhealing scalp masses secondary to trauma. Surgical pathology of Patient A demonstrated biphasic cytology with moderate to poorly differentiated squamous carcinoma connected to the epidermis and a pleomorphic spindled and histiocytoid cell population significant for sarcomatous component. The sarcomatous areas were strongly positive for CD68 and CD163. IHC was positive for keratins, CK5/6, $\mathrm{AE} 1 / \mathrm{AE} 3$, as well as $\mathrm{p} 63$ in the epithelioid component with variable weaker positivity p63 in sarcomatoid cells. This is in agreement with past reports that highlight co-expression of p63 in epithelial and spindle cells [5]. Similarly, surgical pathology for Patient B showed IHC positive vimentin and smooth muscle actin and complete negativity for cytokeratins and p63, suggesting a more complete sarcomatous transformation. The loss of epithelial phenotype including cytokeratin AE1/AE3 and replacement by pleomorphic or spindle morphology accompanied by gain of vimentin expression has been previously described by Brasanac et al. [15].

Interestingly, the type and ratio of sarcomatous elements do not influence prognosis; rather, survival may depend more on the epithelial component. When the squamous cell component concentrations are greater, metastatic rates have been reportedly higher ( $12 \%-50 \%)$ when compared to basal cell components (2\%) $[5,6,16]$. Of the 47 cases analyzed in a report by Zbacnik et al., there was only one metastatic case of axillary basal cell carcinosarcoma to the lung. Furthermore, a recent study of 11 cutaneous carcinosarcomas by Kwak et al reported three cases of metastases to local lymph nodes, two of which were SCC and one keratoacanthoma; however, they did not identify a significant relationship between primary tumor location or size due to a limited sample [17]. Based on these observations, in our report, Patient A had a more lethal form of carcinosarcoma due to the squamous cell component compared to Patient B with a basaloid component. The outcomes of the two patients in our series were contrary to general prognostic factors, as Patient B presented earlier with a less aggressive histologic form of carcinoma and was without distant metastases, but ultimately succumbed to her disease. Along with Bourgeault et al., we agree that a cutaneous carcinosarcoma of the basaloid subtypes should be treated as a high-risk basal cell carcinoma [18]. In both cases, our patients presented later in the disease stage in part due to the negligence of nonhealing wounds, further emphasizing the need for increased awareness of such conditions.

As previously reported, carcinosarcomas may present in various shapes and sizes; therefore, management depends on clinical and histological features [8]. There has been no consensus on definitive margins for excision for cutaneous primary manifestations, as reports describe a range from $0.5-3.5 \mathrm{~cm}$ margins $[5,9,19,20]$. As these defects can be large and in a prominently visible location, it is important for dermatologists to consider referral to plastic surgery for adequate reconstruction. Reconstruction follows traditional teaching in plastic and reconstructive surgery utilizing the reconstructive ladder and/or elevator, ranging from healing by secondary intent up through free-tissue transfer. Depending on the location, size of the defect, and extent of devoid tissue and type, an adequate reconstruction can be as important as the cancer resection to protect any vital structures that may have been exposed. In Patient A, bone deficient of pericranium was exposed in the $5 \times 5 \mathrm{~cm}$ defect. Therefore, a well-vascularized soft tissue rearrangement in the form of an ortichochea flap was an appropriate reconstruction. In Patient B, the wound was significantly larger $(12 \times 7 \mathrm{~cm})$ and extended transcranially, thus exposing bone denuded of pericranium and brain lacking meninges. In this instance, no local options were available to fill the defect and the most appropriate reconstruction included utilizing a thin and pliable anterior lateral thigh free-tissue transfer.

Cutaneous carcinosarcomas may be underreported due to lack of awareness, from both a patient and clinician perspective, and can ultimately prove fatal [16]. Fortunately, unlike skin adnexal and visceral carcinosarcomas, epidermal derived carcinosarcomas may be more visible leading to earlier detection, adequate management, and follow-up. Nevertheless, with cutaneous carcinosarcoma seldom reported in the literature, it is our hope that sharing our experience will not only add to the database of published literature, but also raise awareness of extra-visceral manifestations, and allow for earlier diagnosis and treatment. 


\section{Conclusions}

Compared to visceral counterparts, cutaneous carcinosarcomas have more favorable outcomes due to low metastatic rates likely due to earlier detection, but delayed presentation can be fatal. Nonhealing wounds of the scalp should be properly investigated for potential underlying malignancy. With cutaneous manifestations of carcinosarcoma seldom reported in the literature, it is our hope that reporting unusual instances such as this will raise awareness and allow for earlier diagnoses, treatments, and reconstructions.

\section{Additional Information \\ Disclosures}

Human subjects: Consent was obtained by all participants in this study. Conflicts of interest: In compliance with the ICMJE uniform disclosure form, all authors declare the following: Payment/services info: All authors have declared that no financial support was received from any organization for the submitted work. Financial relationships: All authors have declared that they have no financial relationships at present or within the previous three years with any organizations that might have an interest in the submitted work. Other relationships: All authors have declared that there are no other relationships or activities that could appear to have influenced the submitted work.

\section{References}

1. Quay SC, Harrist TJ, Mihm Jr MC: Carcinosarcoma of the skin. J Cutan Pathol. 1981, 8:241-246. 10.1111/j.1600-0560.1981.tb01004.x

2. Singh R: Review literature on uterine carcinosarcoma . J Cancer Res Ther. 2014, 10:461-468. 10.4103/09731482.138197

3. Upjohn E, Braue A, Ryan A: Primary cutaneous carcinosarcoma: dermoscopic and immunohistochemical features. Australas J Dermatol. 2010, 51:26-28. 10.1111/j.1440-0960.2009.00587.x

4. Rossi G, Cavazza A, Sturm N, et al.: Pulmonary carcinomas with pleomorphic, sarcomatoid, or sarcomatous elements: a clinicopathologic and immunohistochemical study of 75 cases. Am J Surg Pathol. 2003, 27:311324. 10.1097/00000478-200303000-00004

5. Zbacnik AP, Rawal A, Lee B, Werling R, Knapp D, Mesa H: Cutaneous basal cell carcinosarcoma: case report and literature review. J Cutan Pathol. 2015, 42:903-910. 10.1111/cup.12578

6. Tran TA, Muller S, Chaudahri PJ, Carlson JA: Cutaneous carcinosarcoma: adnexal vs. epidermal types define high- and low-risk tumors. Results of a meta-analysis. J Cutan Pathol. 2005, 32:2-11. 10.1111/j.03036987.2005.00260.x

7. Bigby SM, Charlton A, Miller MV, et al.: Biphasic sarcomatoid basal cell carcinoma (carcinosarcoma): four cases with immunohistochemistry and review of the literature. J Cutan Pathol. 2005, 32:141-147. 10.1111/j.0303-6987.2005.00276.x

8. Clark JJ, Bowen AR, Bowen GM, et al.: Cutaneous carcinosarcoma: a series of six cases and a review of the literature. J Cutan Pathol. 2017, 44:34-44. 10.1111/cup.12843

9. Wollina U, Riedel I, Abushika MR, Lotti T, Tchernev G: Giant Pendulous Carcinosarcoma - Squamous Cell Carcinoma-Type - of the Leg - A Case Report and Review of the Literature. Open Access Maced J Med Sci. 2018, 6:112-114. 10.3889/oamjms.2018.036

10. Luong TMH, Akazawa Y, Mussazhanova Z, et al.: Cutaneous pilomatrical carcinosarcoma: a case report with molecular analysis and literature review. Diagn Pathol. 2020, 15:7. 10.1186/s13000-020-0925-y

11. Gates GA, Nguyen J, Binder SW: Rare case report of pilomatrical carcinosarcoma in a pediatric patient. Am J Dermatopathol. 2020, 42:208-210. 10.1097/DAD.0000000000001517

12. Colston J, Hodge K, Fraga GR: Trichoblastic carcinosarcoma: an authentic cutaneous carcinosarcoma. BMJ Case Rep. 2016, 2016:bcr2016214977. 10.1136/bcr-2016-214977

13. McDaniel B, Badri T: Basal Cell Carcinoma. StatPearls. Treasure Island. 2020,

14. Rose RF, Merchant W, Stables GI, Lyon CL, Platt A: Basal cell carcinoma with a sarcomatous component (carcinosarcoma): a series of 5 cases and a review of the literature. J Am Acad Dermatol. 2008, 59:627-632. 10.1016/j.jaad.2008.05.035

15. Brasanac D, Boricic I, Todorovic V, Tomanovic N: Primary cutaneous carcinosarcoma: case report with expanded immunohistochemical analysis. Int J Dermatol. 2008, 47:496-501. 10.1111/j.13654632.2008.03427.x

16. Syme-Grant J, Syme-Grant NJ, Motta L, Stevenson JH, Evans AT: Are primary cutaneous carcinisarcomas underdiagnosed? Five cases and a review of the literature. J Plast Reconstr Aesthet Surg. 2006, 59:14021408. 10.1016/j.bjps.2005.12.057

17. Kwak HB, Park J, Kim HU, Nam KH, Yun SK: Cutaneous carcinosarcoma: a clinicopathologic and immunohistochemical analysis of 11 Korean cases. J Korean Med Sci. 2019, 34:e5. 10.3346/jkms.2019.34.e5

18. Bourgeault E, Alain J, Gagne E: Primary cutaneous carcinosarcoma of the basal cell subtype should be treated as a high-risk basal cell carcinoma. J Cutan Med Surg. 2015, 19:407-411. 10.1177/1203475415575233

19. Suzuki H, Hashimoto A, Saito R, Izumi M, Aiba S: A case of primary cutaneous basal cell carcinosarcoma . Case Rep Dermatol. 2018, 10:208-215. 10.1159/000492525

20. Chittari K, Birnie AJ, Kulkarni KR, Perks AG, Varma S: Sarcomatoid carcinoma of the hand: a clinical case with an aggressive and uncommon presentation. Clin Exp Dermatol. 2012, 37:505-508. 10.1111/j.13652230.2011.04277.x 